\title{
High Speed Differential Drive Mobile Robot Path Following Control With Bounded Wheel Speed Commands
}

\author{
Giovanni Indiveri and Andreas Nüchter and Kai Lingemann
}

\begin{abstract}
The great majority of path following control laws for either kinematical or dynamical mobile robot models are designed assuming ideal actuators, i.e. assuming that any commanded velocity or torque (in the kinematical and dynamical cases respectively) will be instantly implemented regardless of its value. Real actuators are far from being ideal. In particular, only bounded velocities and torques can be realized for any given command. With reference to the kinematical model of a differential drive mobile robot, a known path following control law is modified to account for actuator velocity saturation. The proposed solution is experimentally shown to be particularly useful for high speed applications where accounting for actuator velocity saturation may have a large influence on performance.
\end{abstract}

\section{INTRODUCTION}

In the last few years tremendous progress in mobile robot motion control has been achieved. Typical problems addressed in literature include point stabilization, trajectory tracking and path following [3] for which either kinematic or dynamic solutions are derived. In real implementations it is important that the controller outputs are bounded to prevent hardware damages. When actuator bounds are not explicitly taken into account during the control design phase, a common practical solution is to artificially saturate the actuator inputs (i.e. the controller outputs) to their upper bounds at cost of performance. This paper proposes a path following control law that takes actuator velocity bounds explicitly into account. The resulting solution appears to be particularly well suited for high speed path following applications.

Given a curve $l \in R^{p}$ (where $p=2$ or 3 ) parametrized by some scalar $s \in R$ (by example the curvilinear abscissa), denoting with $r \in R^{q}$ the pose (position and orientation) of the vehicle being

$$
\dot{r}=f(r, \xi, u) \quad: \quad u \text { control input }
$$

( $\xi$ non manipulable input) and with $d(r, l) \in R$ a suitable distance between the vehicle and the path, the path following control law design problem consists in finding a function $u=u^{*}(r, l)\left(u^{*}\right.$ may eventually depend by $\xi$ too) such that

$$
\lim _{t \rightarrow \infty} d\left(\left.r\right|_{u=u^{*}}, l\right)=0 .
$$

G. Indiveri is with Dipartimento Ingegneria Innovazione, University of Lecce, Italy. giovanni.indiveri@unile.it

Andreas Nüchter and Kai Lingemann are with the Knowledge Systems Research Group of the Institute of Computer Science, University of Osnabrück, Germany. \{nuechter, lingemann\} @ informatik.uni-osnabrueck.de
Indeed most often the design procedure aims at making the origin an asymptotically (possibly globally) stable equilibrium point for the dynamics of $d\left(\left.r\right|_{u=u^{*}}, l\right)$. This design technique will guarantee not only closed loop convergence of $d(r, l)$ to zero (as required by (2)), but also its stability implying a certain degree of robustness to model uncertainty and measurement noise. In mobile robotics applications $p$ is usually assumed to be 2 (planar case) whereas the dimension of the state variable $q$ depends on whether a kinematical or dynamical robot model is used to design the controller. Although from the perspective of physics the system model is obviously always dynamical, dynamics may be negligible if the actuators should be able to produced much larger accelerations than the desired ones. In this case, a closed loop controller may be designed on the basis of a kinematical robot model: The system inputs (i.e. the controller outputs $u^{*}$ ) would be the actuator velocities $\dot{q}_{i}$ and the system outputs would be robot velocities and their integrals. In practice, the kinematically computed actuator velocities $u^{*}=\dot{q}_{i}$ will be used as reference values for the (fast) actuator dynamics: If the lag between such reference values and the actuator produced velocities should be sufficiently small (with respect to smallest time constant associated to the spectrum of $\ddot{q}_{i}$ ), the kinematically designed control law will perform well. When the actuator dynamics should not be much faster than the desired closed loop system dynamics, it could not be neglected and the control law would need to be designed on the basis of a full dynamic model. In short, the choice of using a kinematical or dynamical vehicle model to design the controller depends on the actuator dynamics as compared to the desired closed loop one. If, by example, a mobile robot should be equipped with high torque / low speed motors able to produce high accelerations as compared to the desired ones, a kinematical based path following design coupled with a sufficiently fast and accurate lower level speed motor controller could be proper. If, instead, low torque / high speed motors should be employed, a full dynamic model based design would need to be considered. It should be noticed that the path following control design problem has been solved for either the dynamical or kinematical case, but usually assuming ideal actuators. Namely any commanded torque (in the dynamic case) or speed (in the kinematical case) is assumed to be instantly implemented regardless of its value. Real actuators are far from being ideal and will exhibit saturation in case the commanded signals should exceed given thresholds. In this paper the case of kinematical path following control for a differential drive mobile robot is considered, but taking explicitly into account actuator satura- 


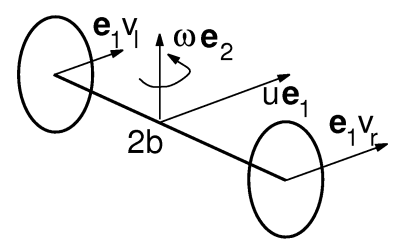

Fig. 1. Differential drive

tion limits. The problem at hand is particularly relevant when mobile robots equipped with high torque (eventually due to high gear ratio) motors are considered. In order to drive such systems at high speeds without the risk of performance degradation due to saturating wheel speeds, saturation needs to be explicitly taken into account in the control law. The proposed solution builds on the path following controller described in [10].

The robot model is described in Section II while the path following algorithm is derived and analyzed in Section III. Experimental results are presented in Section IV. Conclusions and future work are briefly discussed in Section V.

\section{RoBOt MODEL}

Consider a differential drive vehicle having two independently actuated wheels on a common axis at a distance $2 b$ one from the other. Call $u \mathbf{e}_{1}$ and $\omega \mathbf{e}_{2}$ the linear and angular velocities of the mid point of such axis being $\mathbf{e}_{1}$ and $\mathbf{e}_{2}$ body fixed unit vectors as depicted in Fig. 1. Call $\mathbf{e}_{1} v_{r}$ and $v_{l} \mathbf{e}_{1}$ the velocities of the center of the right and left wheels that are assumed to roll perfectly. The kinematic model linking the scalars $u, \omega, v_{r}$ and $v_{l}$ is:

$$
\begin{aligned}
u & =\frac{1}{2}\left(v_{r}+v_{l}\right) \\
\omega & =\frac{1}{2 b}\left(v_{r}-v_{l}\right)
\end{aligned}
$$

where $\left|v_{r}\right|$ and $\left|v_{l}\right|$ will be bounded by some given value $V_{m}$. As known, a differential drive vehicle modeled by equations (3-4) can move on paths of arbitrary curvature $\kappa$, as

$$
\kappa=\frac{1}{b} \frac{v_{r}-v_{l}}{v_{r}+v_{l}} .
$$

Indeed for any $v_{l}=-v_{r}$ the corresponding path curvature would be infinite, i.e. the vehicle would turn on the spot. Arbitrarily large curvature values can be implemented if the wheels rotate in opposite directions. Yet if the vehicle should be required to move at high linear speeds, commanding wheel speeds of different sign in order to make sharp turns should be avoided in practice in order not to overstress the electromechanical structures (gear boxes, tires, DC motor $\mathrm{H}-$ Bridge power circuits) of the robot. If the wheel speeds $v_{l}$ and $v_{r}$ should be constrained to positive values only, than the curvature $\kappa$ given by equation (5) would be bounded

$$
\kappa \in\left[-\frac{1}{b}, \frac{1}{b}\right] \quad \text { if } v_{l}, v_{r} \in\left[0, V_{m}\right]
$$

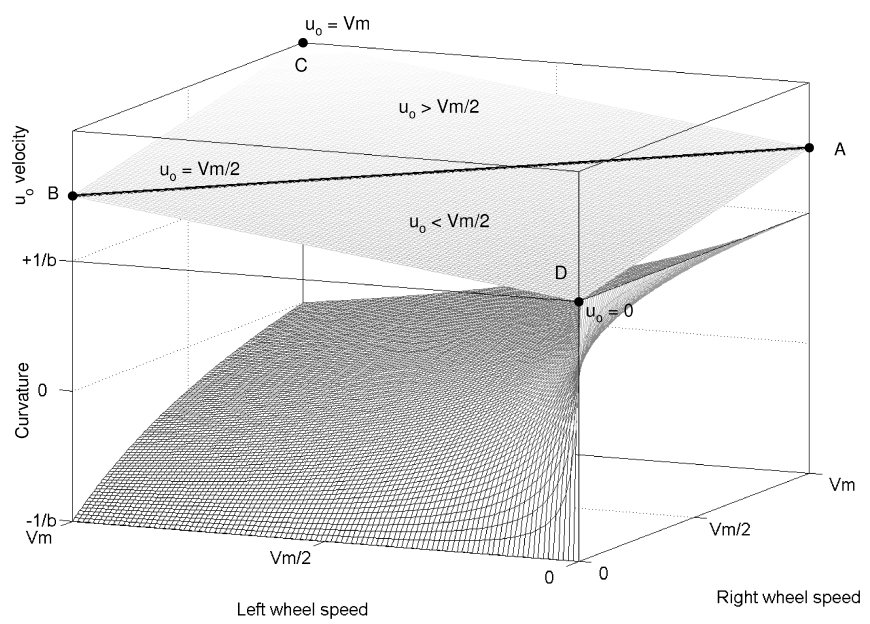

Fig. 2. Differential drive curvature and total linear velocity as functions of the wheels speeds.

and the linear speed $u>0$ should be constrained to $u \leq$ $V_{m} / 2$ to let $\kappa$ span its full range $[-1 / b, 1 / b][6]$.

The plots of the curvature (5) and of the linear velocity $u(t)=u_{o}$ (constant) (3) with respect to $v_{l}, v_{r} \in\left[0, V_{m}\right]$ are reported in Fig. 2 on a common vertical axis. The bottom part of the $\mathrm{z}$-axis (from point $-1 / b$ to $1 / b$ ) refers to curvature whereas the upper part (from point $1 / b$ to the upper end of the scale) refers to the $u(t)$ velocity. With reference to the $u(t)$ plane depicted in the upper part of the plot, point $D$ corresponds to $u_{o}=0$, point $C$ to $u_{o}=V_{m}$ and the line $B A$ corresponds to $u_{o}=V_{m} / 2$. All points belonging to lines parallel to $B A$ refer to constant values of $u_{o}$, in particular all points in the region $\{B A D\}$ refer to linear vehicle velocities in $\left[0, V_{m} / 2\right]$ and all points in $\{B A C\}$ refer to linear vehicle velocities in $\left[V_{m} / 2, V_{m}\right]$. Points on the (non depicted) line $D C$ from $D$ to $C$ refer to increasing linear speeds with zero curvature (i.e. angular velocity) due to the fact that the two wheels have the same speed. The picture shows that in order to exploit the full range $[-1 / b, 1 / b]$ of possible curvatures at all times with the constraints that $0 \leq v_{l} \leq V_{m}$ and $0 \leq v_{r} \leq V_{m}$, the linear speed $u(t)$ of a differential drive vehicle should always be smaller or equal to $V_{m} / 2$. Notice that this may be a rather conservative bound as if the reference path should be a straight line $(\kappa=0)$, the maximum feasible linear velocity would be of course $V_{m}$ corresponding to $v_{l}=v_{r}=V_{m}$. Notice that in the above hypothesis that the wheels of differential drive robot turn in one direction only (i.e. $v_{l} \geq 0, v_{r} \geq 0$ ), given that the resulting admissible curvature is bounded $(|\kappa| \leq 1 / b)$, in any path following application the reference path curvature $\kappa_{r}$ will need to be bounded too. In the sequel, the upper bound on the absolute value of the reference path curvature will be denoted with $\kappa_{r \max }>0$ and it will be assumed that

$$
\left|\kappa_{r}\right|<\kappa_{r \max }<1 / b
$$




\section{Path Following Control}

Consider the path following controller designed in [10]: given a Serret-Frenet frame $\{F\}$ moving along the planar path, call $P$ its origin having curvilinear abscissa $s$ with respect to an arbitrary path point (origin of the curvilinear abscissa). Call $\{I\}$ a fixed inertial frame and $Q$ the mid point of the differential drive robot axis such that $Q$ has coordinates $\left(s_{1}, y_{1}\right)$ in $\{F\}$ and $(x, y)$ in $\{I\}$ (refer to figure (cf. Fig. 3). Calling $\kappa_{r}$ the curvature of the reference path, the kinematics of $Q$ in $\{I\}$ would be given by the unicycle model

$$
\begin{aligned}
\dot{x} & =u \cos \theta_{m} \\
\dot{y} & =u \sin \theta_{m} \\
\dot{\theta}_{m} & =\omega
\end{aligned}
$$

and by

$$
\begin{aligned}
\dot{s}_{1} & =-\dot{s}\left(1-\kappa_{r} y_{1}\right)+u \cos \theta \\
\dot{y}_{1} & =-\kappa_{r} \dot{s} s_{1}+u \sin \theta \\
\dot{\theta} & =\omega-\kappa_{r} \dot{s}
\end{aligned}
$$

in $\{F\}$ (refer to [10] for details) being $\theta_{m}$ and $\theta$ the vehicle's heading in $\{I\}$ and $\{F\}$ respectively. Following [10], consider the Lyapunov candidate function

$$
V_{1}=\frac{1}{2}\left(s_{1}^{2}+y_{1}^{2}\right)+\frac{1}{2 \gamma}\left(\theta-\delta\left(y_{1}, u\right)\right)^{2}
$$

for some positive $\gamma$. In the assumptions that $i$ ) $\left.\lim _{t \rightarrow \infty} u(t) \neq 0, \quad i i\right) \delta(0, u)=0$ and $\left.i i i\right)$ $y_{1} u \sin \delta\left(y_{1}, u\right) \leq 0 \quad \forall y_{1}, u$ the time derivative of $V_{1}$ can be made globally semi-negative definite by the choice

$$
\begin{aligned}
& \dot{\theta}=\dot{\delta}-\gamma y_{1} u \frac{\sin \theta-\sin \delta}{\theta-\delta}-k_{2}(\theta-\delta) \\
& \dot{s}=u \cos \theta+k_{1} s_{1} \quad: \quad k_{1}>0, k_{2}>0
\end{aligned}
$$

that implies

$$
\frac{d V_{1}}{d t}=-k_{1} s_{1}^{2}-\frac{1}{\gamma}(\theta-\delta)^{2}+y_{1} u \sin \delta \leq 0 .
$$

Closed loop asymptotic global stability of the equilibrium $\left(s_{1}, y_{1}, \theta\right)=(0,0,0)$ can be shown by invoking LaSalle's Invariant Set Theorem or Barbalat's Lemma [7] to the above Lyapunov candidate function and to the closed loop state

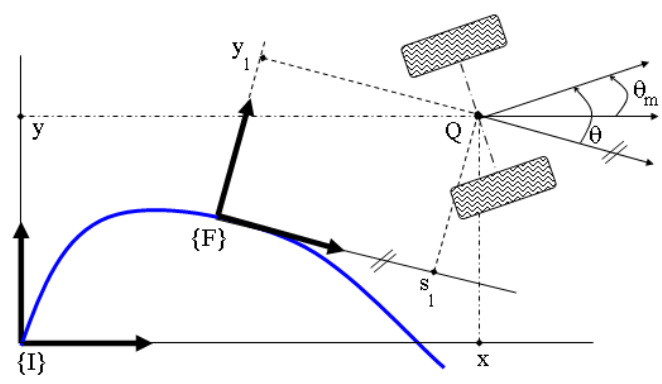

Fig. 3. The used Frenet frame. equations. Notice that as already pointed out in [1], global stability and convergence of the path following error to zero can be achieved by exploiting the extra degree of freedom provided by $\dot{s}$. Alternative solutions as the by now classical one described in [3] guarantee only local stability of the error to zero and require rather stringent initial conditions on the system's state to avoid kinematical singularities that are absent in the model used in [10] and in this paper. Also notice that the function $\delta\left(y_{1}, u\right)$ introduced in equation (14) has the only purpose of shaping the transient convergence of the state to zero and should thus be regarded as an extra design degree of freedom. Possible choices for $\delta$ include $\delta:=0$ or $\delta\left(y_{1}, u\right)=\operatorname{sign}(u) k_{3} \tanh \left(y_{1}\right)$ for some nonnegative $k_{3}$ as suggested in [10]. In the sequel it will be assumed that $\delta\left(y_{1}, u\right)$ does not depend on $|u|$, namely that $\delta$ is invariant with respect to the absolute value of the robot's linear velocity.

By combining equations (3), (4), (13), (15) and (16) the commanded wheel speeds result in

$$
\begin{aligned}
& v_{r}=u+b\left(\kappa_{r} \dot{s}+\dot{\theta}\right) \\
& v_{l}=u-b\left(\kappa_{r} \dot{s}+\dot{\theta}\right)
\end{aligned}
$$

As reported in [10], the control law given by equations (15) and (16) guarantees all the state variables to remain bounded. Notice that replacing equations (18) and (19) in (5) the closed loop curvature of the vehicle results in

$$
\kappa_{\text {closed loop }}=\frac{\kappa_{r} \dot{s}+\dot{\theta}}{u} .
$$

In the light of the above and of section II, in order for the path following problem to be solved with the constraints that $v_{l}, v_{r} \in\left[0, V_{m}\right]$, it must be assumed that the gain constants in equations (18) and (19), the robot initial conditions and the reference path are such that the absolute value of the closed loop curvature given by equation (20) is always bounded by $1 / b$. This assumption will be considered satisfied throughout the remaining of the paper. This implies, in particular, that the reference curvature $\kappa_{r}$ should be bounded as already noticed in section II. Without entering in the details of the analysis for the sake of brevity, it should be noticed that the closed loop curvature can be written as:

$$
\begin{aligned}
\kappa_{\text {closed loop }}= & \kappa_{r} \cos \theta+T(\cdot) \\
T:= & \frac{1}{u}\left(k_{r} k_{1} s_{1}+\dot{\delta}-k_{2}(\theta-\delta)+\right. \\
& \left.-\gamma y_{1} u \frac{\sin \theta-\sin \delta}{\theta-\delta}\right)
\end{aligned}
$$

where as long as $u \in\left[V_{m} / 2, V_{m}\right]: V_{m}>0$ the term $T(\cdot)$ can be made arbitrarily small for any initial condition by acting on the design terms $\delta, \gamma, k_{1}$ and $k_{2}$. In particular such design terms need to be selected such that $|T(\cdot)| \leq 1 / b-\kappa_{r \max }$ in order to guarantee that $\left|\kappa_{\text {closed loop }}\right| \leq 1 / b$. Hence the above hypothesis on the boundedness of the closed loop curvature is not particularly restrictive. Moreover being $(0,0,0)^{T}$ a 
(closed loop) globally asymptotically stable equilibrium for the path following error $\left(s_{1}, y_{1}, \theta\right)^{T}$, notice that

$$
\lim _{t \rightarrow \infty} \kappa_{\text {closed loop }}=\kappa_{r} .
$$

Within the described setting, in order to command wheel velocities (18-19) corresponding to the highest possible (positive) linear velocity $u$, the following should be satisfied:

$$
\begin{aligned}
& \max \left\{v_{l}, v_{r}\right\}=|u|\left(1+b\left|\kappa_{\text {closed loop }}\right|\right)=V_{m} \Longrightarrow \\
& u=\frac{V_{m}}{\left(1+b\left|\kappa_{\text {closed loop }}\right|\right)} .
\end{aligned}
$$

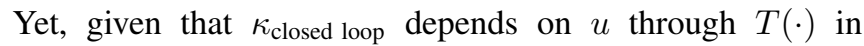
equation (21), equation (23) cannot be used to compute $u$. As a consequence, given the analysis carried out in section II, in the stated hypothesis that $\left|\kappa_{\text {closed loop }}\right| \leq 1 / b$, that $\left|\kappa_{r}\right|<\kappa_{r \max }<1 / b$, and in the light of the fact that $\kappa_{\text {closed loop }} \longrightarrow \kappa_{r}$ as the path following error tends to zero, the following strategy is proposed:

$$
u=\left\{\begin{array}{lll}
V_{m} / 2 & \text { if } & V_{1} \geq \varepsilon \\
V_{m} /\left(1+b\left|\kappa_{r}(s)\right|\right) & \text { if } & V_{1}<\varepsilon
\end{array}\right.
$$

being $\varepsilon>0$ a design threshold parameter to be determined based upon the reference path and the initial robot conditions. The rationale behind equation (24) is that the linear speed $u$ should be allowed to take values larger than $V_{m} / 2$ only when the path following error (as measured by the Lyapunov function $V_{1}$ ) is small and hence $\kappa_{\text {closed loop }}$ tends to $\kappa_{r}$. In this limit, the value of $u$ given by equation (24) when $V_{1}<\varepsilon$ tends to the optimal value given by equation (23). In practical applications, the $\varepsilon$ parameter can be tuned by monitoring the closed loop value of $\max \left\{\left|v_{l}\right|,\left|v_{r}\right|\right\}$ : in particular, for vanishing $\varepsilon$ the maximum wheel speed $\max \left\{\left|v_{l}\right|,\left|v_{r}\right|\right\}$ should remain bounded by $V_{m}$. Persistent values of $\max \left\{\left|v_{l}\right|,\left|v_{r}\right|\right\}$ significantly below the $V_{m}$ threshold suggest that $\varepsilon$ may be increased.

\section{EXPERIMENTAL RESULTS}

The following section describes preliminary experiments to test the control law.

\section{A. The Mobile Robot Kurt3D}

In our experiments the skid steered mobile robot Kurt3D has been used. Kurt3D is based on a KURT2 mobile robot platform that is available with different wheels, motors and gear boxes. The outdoor version (Fig. 4 left) has six $16 \mathrm{~cm}$ wheels, where the two center wheels are shifted sideways/outwards to shorten the overall length of the robot. With its two $90 \mathrm{~W}$ motors that power the six wheels the robot reaches a maximal velocity of $1.1 \mathrm{~m} / \mathrm{s}$. The high speed indoor version (Fig. 4 right) drives with the same motors and a different, low torque gear box up to $4.1 \mathrm{~m} / \mathrm{s}$. On both vehicles types front and rear wheels have no tread pattern to enhance rotation. Therefore, the differential drive model is applicable. The robot has a C-167 microcontroller and two Centrino laptops for sensor data acquisition and transmission.

To cope with the different characteristics of the KURT2 robots, we have implemented a motor controller that consists
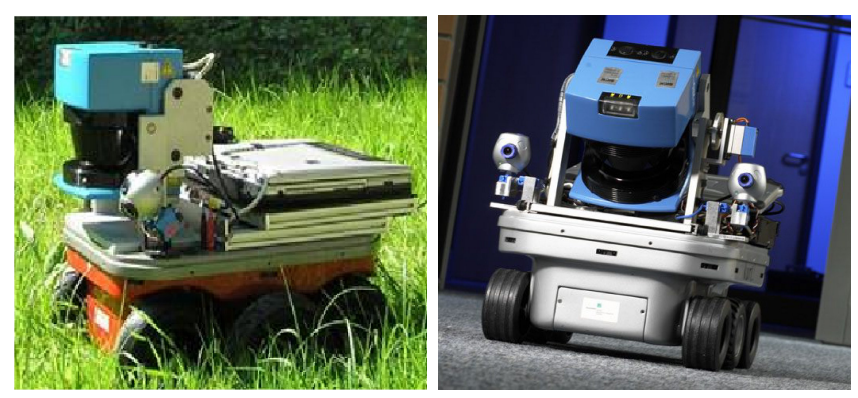

Fig. 4. KURT2 variants. Slow outdoor (left) and high speed indoor Kurt3D.

of a feed forward PI controller combined with a lookup table for mapping speed to PWM values and linearizing the motor signal. It combines open and closed loop control concepts and ensures a fast response time with only a small overshoot. Details of the low level control can be found in [8].

\section{B. Following a Circular Curve}

In order to evaluate the performance of the proposed control law, we first used a circular curve as path to follow. In an initial experiment, a test without load has been performed, i.e., the robot wheels had no contact with the ground. Fig. 5 shows the path the robot would have driven, based on wheel encoder measurements only. To test the path following, we applied two kind of perturbations to this no load operation: First, we stopped one wheel manually and second, we put additional acceleration on one wheel by turning it faster than the controlled speed. Top priority is to keep the vehicle on the path, thus the second wheel stops or accelerated accordingly (cf. Fig. 5, bottom).

Exact path following is only possible if and only if the self localization of the robot is precise. Exact and continuous pose estimation is the key for real applicability of the proposed controller. Kurt3D's odometry and gyro measurements take place with a frequency of $100 \mathrm{~Hz}$, whereas two dimensional laser range scans are taken with $75 \mathrm{~Hz}$. Fusing odometry with the gyro data is done by the following deterministic method: If a rotation is measured, the gyro value is taken [11]. Otherwise the drift $e$ is learned with the rate $\lambda$ :

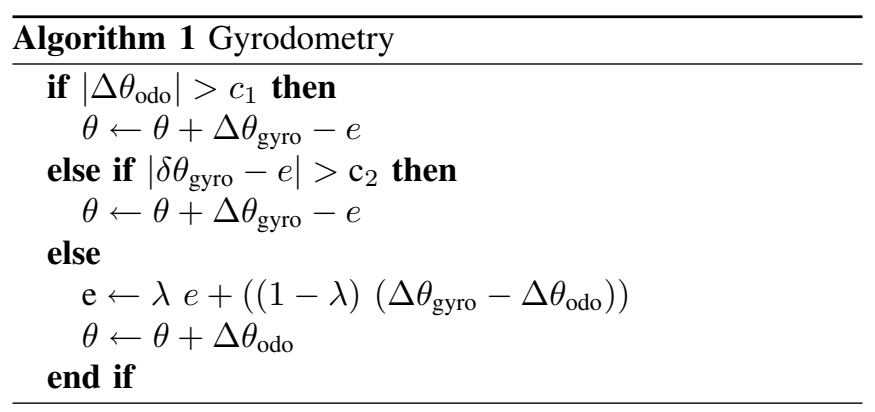

In addition to correcting the robot pose with the gyro, the scanner data is used. The well known ICP algorithm is applied to correct the data [2], [9]. The ICP algorithm 
Odometry based path following (no load)
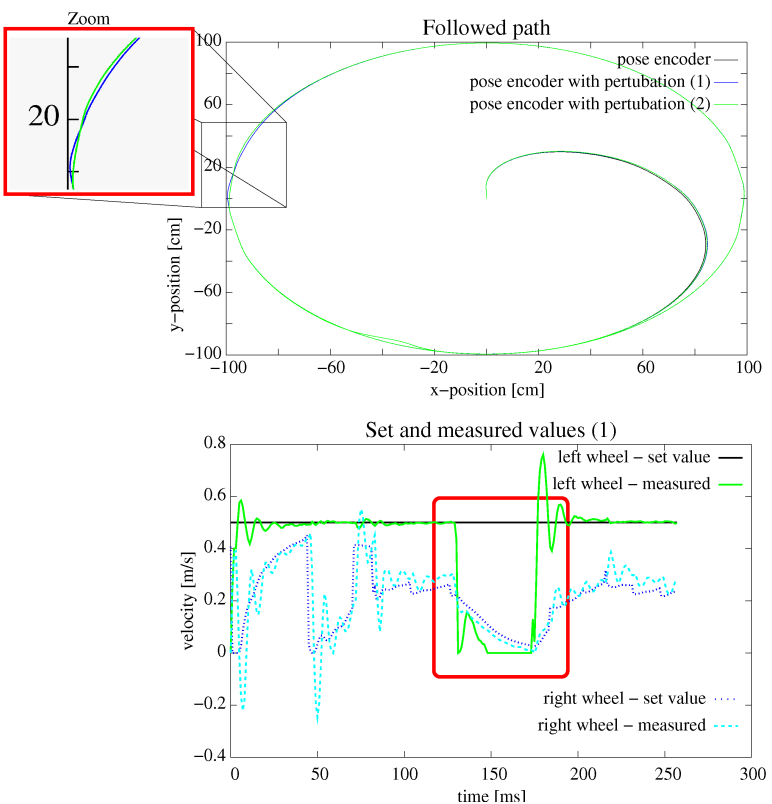

Fig. 5. Following a circular path in no load operation with applied perturbations: Top: Followed path: In (1) one wheel has been manually stopped, and in (2) one wheel was accelerated. Bottom: When one wheel is stopped, the second wheel stops instantly too, to keep the vehicle on the given path. Thus, the path following is stable.

calculates iteratively point correspondences between two scans $M$ and $D$. In each iteration step, the algorithm selects the closest points as correspondences and calculates the transformation $(\mathbf{R}, \mathbf{t})$ that minimizes the equation

$$
E(\mathbf{R}, \mathbf{t})=\sum_{i=1}^{N_{m}} \sum_{j=1}^{N_{d}} w_{i, j}\left\|\mathbf{m}_{i}-\left(\mathbf{R d}_{j}+\mathbf{t}\right)\right\|^{2}
$$

where $N_{m}$ and $N_{d}$, are the number of points in the so called model set $M$ or the data set $D$, respectively, and $w_{i, j}$ the weights for the point matches. The weights are assigned as follows: $w_{i, j}=1$, if $\mathbf{m}_{i}$ is the closest point to $\mathbf{d}_{j}$ within a close limit, $w_{i, j}=0$ otherwise.

We have implemented $k$ d-tree search for fast closest point computation. However, ICP localization can only be done with a frequency of about $20 \mathrm{~Hz}$. Therefore, we extrapolate the Gyrodometry between two consecutive ICP runs in order to provide the system a continuous control input. Extrapolated pose information are also used as starting guess for minimizing the ICP error function (Eq. (25)).

Fig. 6 shows the resulting trajectories, using the different localization methods, with pure ICP as reference. Nevertheless, the ICP localization might also be erroneous, since small errors accumulate. Solutions with multi-hypotheses methods, e.g., Markov [5] or Monte-Carlo localization [4] are available, but they show difficulties with providing fast control input in not-restricted and unmapped environments.

Finally, Fig. 8 shows the wheel velocities, corresponding to the circular path. While in the original control law saturation is nearly avoided, in the high speed case one wheel
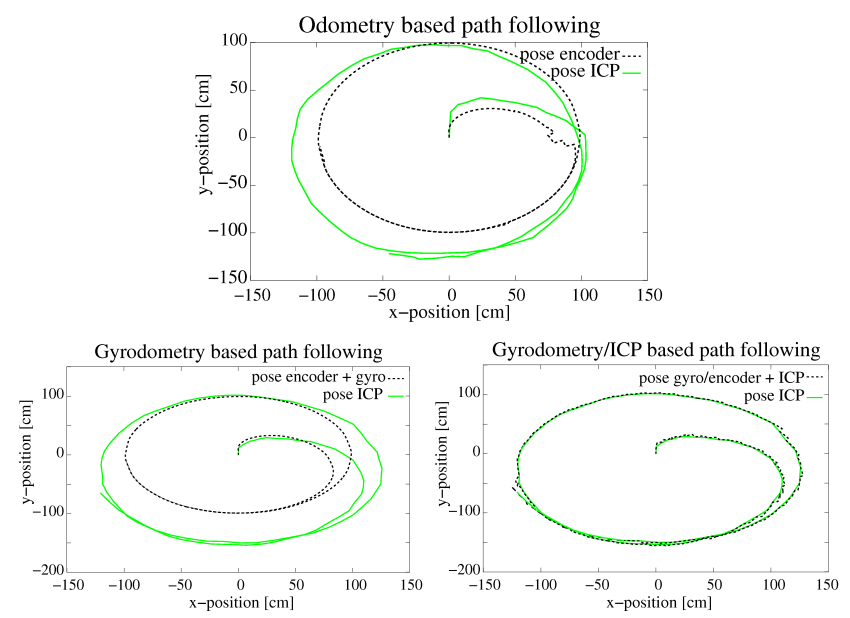

Fig. 6. Following a circular path using different localization methods Top: Odometry based path following. Bottom left: Gyrodometry based path following. Bottom right: Combined ICP/Gyrodometry path following. All paths are compared with ICP localization.

is always assigned the maximal control input, due to the circular path.

\section{Following an Arbitrary Shaped Path}

For an additional test, we used a longer path, that included turns with different radii and straight parts. The path is given in Fig. 7 and the resulting velocities are shown in Fig. 9. The whole path is driven in about $50 \%$ of the time. One wheel is always saturated, on the straight parts quite often both motors.

\section{CONClusion}

Building on the path following control law presented in [10], a kinematic path following control solution taking explicitly into account the maximum feasible wheel speed has been derived for a differential drive robot. Differential drive robots are technically able to drive infinite curvature paths: in particular, in order to drive on paths with curvature $\kappa>1 / b$ being $b$ the intra-wheel distance, the wheels need to spin in opposite directions. As in high speed applications such commands could over stress the electromechanical drive systems (in particular the $\mathrm{H}$ bridge motor amplifiers and the motor gear boxes) and trigger undesired dynamical effects as sliding, it might be desired to avoid them. If the reference path has a maximum curvature bounded by $|\kappa| \leq 1 / b$, the proposed schema guarantees globally converging path following error to zero while driving at the maximum possible linear speed. Preliminary experimental results are reported validating the proposed solution.

In future work we are going to concentrate on improving the robot's self localization to ensure fast, exact and reliable pose and velocity estimates as input for the proposed controller, since the control quality depends crucially on these parameter. We will set up an experiment with an external laser scanner to track the robot path in order to provide ground truth for localization. 


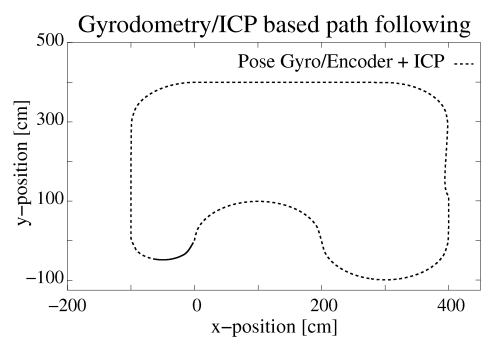

Fig. 7. Following a path with long straight parts and curves with different curvatures.

Finally, we plan to test the high speed path following system in a robotic slalom competition.

\section{ACKNOWLEDGMENTS}

The authors would like to acknowledge the Fraunhofer Institute IAIS, Sankt Augustin, Germany, for the cooperation in developing the initial robot controller. Especially we would like to thank Hartmut Surmann for his patience to discuss controller software. Furthermore, we thank Joachim Hertzberg for supporting our work.

\section{REFERENCES}

[1] M. Aicardi, G. Casalino, A. Bicchi, and A. Balestrino. Closed Loop Steering of Unicyle-Like Vehicles via Lyapunov Techniques. IEEE Robotics and Automation Magazine, 1995.
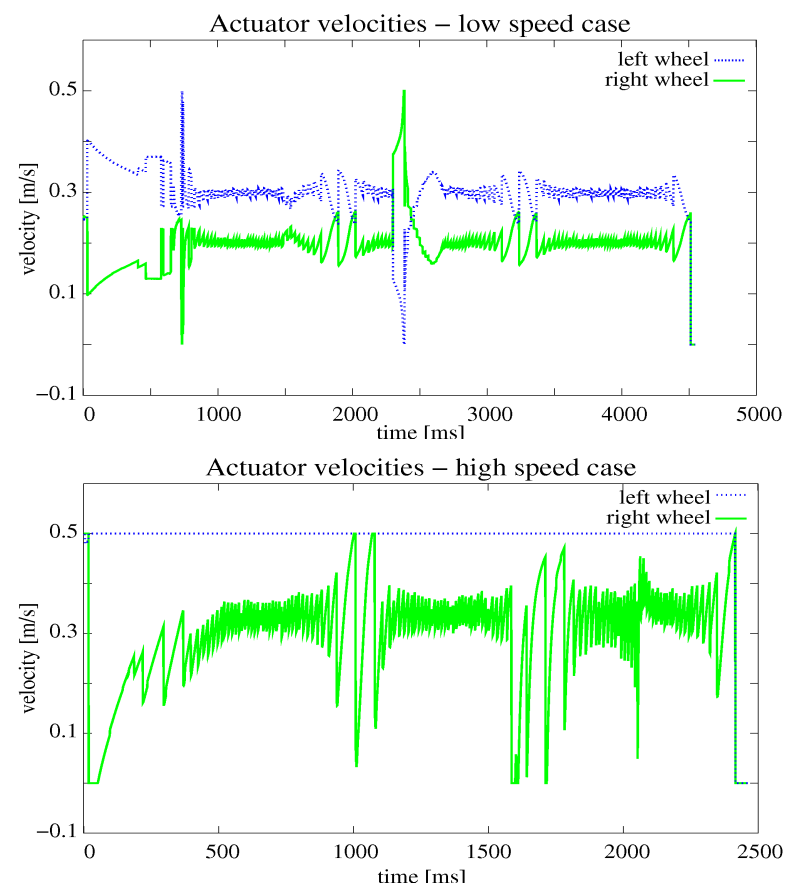

Fig. 8. Comparison of low speed behavior (top) and high speed behavior (bottom), while following a circular line. Note: The $x$-axis (time) is scaled differently.
[2] P. Besl and N. McKay. A method for Registration of 3-D Shapes. IEEE Transactions on Pattern Analysis and Machine Intelligence, 14(2):239 - 256, February 1992.

[3] C. Canudas de Wit, H. Khennouf, C. Samson, and O. Sørdalen. Nonlinear Control Design for Mobile Robots. In Yuan F. Zheng, editor, Recent Trends in Mobile Robots, pages 121 - 156. World Scientific Series in Robotics and Automated Systems, 1993.

[4] D. Fox, W. Burgard, F. Dellaert, and S. Thrun. Monte Carlo Localization: Efficient Position Estimation for Mobile Robots. In Proceedings of the 16th National Conference on Artificial Intelligence (AAAI '99), 1999.

[5] D. Fox, W. Burgard, and S. Thrun. Active markov localization for mobile robots. Journal Robotics and Autonomous Systems, 25:195 207, 1998.

[6] G. Indiveri and M. L. Corradini. Switching linear path following for bounded curvature car-like vehicles. In Proceedings of the IFAC Symposium on Intelligent Autonomous Vehicles (IAV '04), Lisbon, Portugal, July 2004.

[7] H.K. Khalil. Nonlinear Systems. Prentice-Hall, Inc. New Jersey, USA, second edition, 1996.

[8] K. Lingemann, A. Nüchter, J. Hertzberg, and H. Surmann. About the Control of High Speed Mobile Indoor Robots. In Proceedings of the Second European Conference on Mobile Robotics (ECMR '05), pages 218 - 223, Ancona, Italy, September 2005.

[9] F. Lu and E. Milios. Robot Pose Estimation in Unknown Environments by Matching 2D Range Scans. In IEEE Computer Vision and Pattern Recognition Conference (CVPR '94), pages 935-938, 1994.

[10] D. Soetanto, L. Lapierre, and A. Pascoal. Adaptive, Non-Singular Path-Following Control of Dynamic Wheeled Robots. In Proceedings of the 42nd IEEE Conference on Decision and Control (CDC '03), pages 1765 - 1770, Maui, Hawaii U.S.A., December 2003.

[11] E. Solda, R. Worst, and J. Hertzberg. Poor Man's Gyro-based Location. In Proceedings of the 5th IFAC Symposium on Intelligent Autonomous Vehicles (IAV '04), Lisabon, Portugal, July 2004.
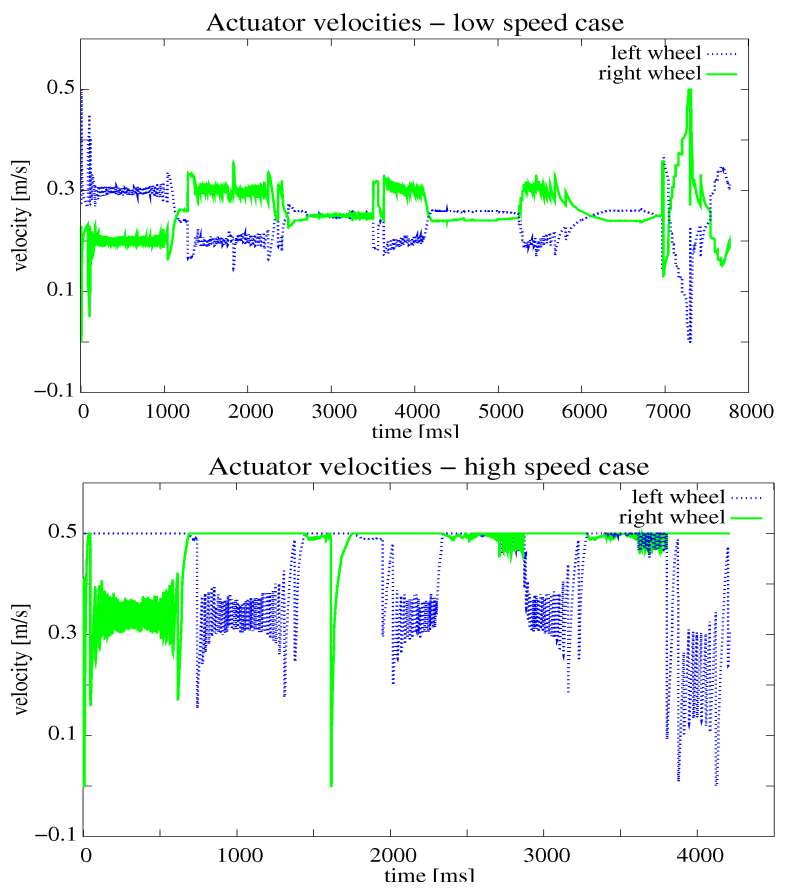

Fig. 9. Comparison of low speed behavior (top) and high speed behavior (bottom), while following a circular line. Note: The $x$-axis (time) is scaled differently. 